\title{
Analytic mode-matching for acoustic scattering in three dimensional waveguides with flexible walls: application to a triangular duct
}

\author{
Jane B Lawrie \\ Department of Mathematics \\ Brunel University, Uxbridge \\ UB8 3PH,UK \\ Jane.Lawrie@brunel.ac.uk
}

\begin{abstract}
An analytic mode-matching method suitable for the solution of problems involving scattering in three-dimensional waveguides with flexible walls is presented. Prerequisite to the development of such methods is knowledge of closed form analytic expressions for the natural fluid-structure coupled waveforms that propagate in each duct section and the corresponding orthogonality relations. In this article recent theory [Lawrie, Proc. R. Soc. A. 465, 2347-2367 (2009)] is extended to construct the non-separable eigenfunctions for acoustic propagation in a three-dimensional rectangular duct with four flexible walls. For the special case in which the duct cross-section is square, the symmetrical nature of the eigenfunctions enables the eigenmodes for a right-angled, isosceles triangular duct with flexible hypotenuse to be deduced. The partial orthogonality relation together with other important properties of the triangular modes are discussed. A mode-matching solution to the scattering of a fluid-structure coupled wave at the junction of two identical semi-infinite ducts of triangular cross-section is demonstrated for two different sets of "junction" conditions.
\end{abstract}

Keywords Three dimensional duct; flexible walls; elastic plate; triangular cross-section; mode-matching; acoustic propagation

\section{Introduction}

The scattering of waves in ducts or channels has long been of interest to scientists and engineers. Analytic mode-matching provides an appealing approach to the solution of many such problems. Traditionally the method has been restricted to canonical geometries in which the boundary value problems involve a governing equation such Laplace's or Helmholtz's and in which the duct/channel walls are described by simple conditions (soft, hard or impedance). The underlying eigen-systems for such boundary value problems are Sturm-Liouville in type and thus have well defined orthogonality properties. For 
more complicated geometries and/or ducts bounded by surfaces described by high-order conditions (such as the thin plate equation) alternative solution methods were necessary [1]-[3]. The past decade has seen a dramatic change in this situation. Hybrid modematching methods have been devised to deal with more complicated geometries [4]-[9] and the theory underpinning wave propagation in two-dimensional (2D) ducts with high order boundary conditions has been extensively developed [10, 11].

Problems involving wave propagation in three dimensional (3D) ducts or channels with flexible walls remain, however, both challenging and of considerable interest to engineers [12]-[17]. Although mode-matching methods generally neglect the effects of break-out, they do enable physical insight into the underlying scattering processes and also provide benchmark solutions for fully numerical approaches. Thus, the development of a hybrid analytic-numerical mode-matching method to address this class of problem would be a significant asset to the scientific community. Recent advances in this respect have been forged by Lawrie [18] who established much of the mathematical theory underlying acoustic propagation in a $3 \mathrm{D}$ rectangular duct with one flexible wall. A second article extends the theory to ducts with porous linings, internal structures or orthotropic boundaries, [19]. For each of the ducts considered in [18]-[19], the "corner conditions" applied along the length of the duct where the flexible wall meets the adjacent rigid wall dictate that the eigenmodes are non-separable in form. As a result only a "partial" orthogonality relation can be constructed and this, of course, complicates the mode-matching procedure. In contrast, Mondal et al [20] consider a 3D problem involving the scattering of flexural gravity waves in a rectangular channel due to a crack in a floating ice sheet. In this case, as in [21], the nature of the "corner conditions" are such that the 3D eigenmodes are separable and, thus, the (generalised) orthogonality relation (OR) is more straightforward to apply.

The aim of this article is to develop a mode-matching approach for 3D ducts with flexible walls and in which the eigenfunctions are non-separable. In section 2, the theory established in [18]-[19] is extended to a rectangular duct in which all four walls are flexible. Symmetry is then used to construct, in section 3, the eigenmodes corresponding to acoustic propagation in a right angled, isosceles triangle in which the hypotenuse comprises a thin elastic plate whilst the other two walls are rigid. The (partial) orthogonality relation and other relevant properties of the eigenmodes are stated. In section 4, a typical problem involving the scattering of an incident fluid-structural mode at the junction two identical semi-infinite ducts of triangular cross-section is considered. The solution to this problem is crucially dependent on the edge conditions applied at the junction of the two plates. It is demonstrated that an analytic mode-matching scheme can be constructed by which to determine the scattered field for two distinct sets of junction conditions. A comprehensive discussion of the method is presented in section 5 . 


\section{Propagation in a 3D duct with four flexible walls}

A 3D duct of rectangular cross-section occupies the region $-\infty<\bar{x}<\infty,-\bar{a} \leq \bar{y} \leq \bar{a}$, $-\bar{b} \leq \bar{z} \leq \bar{b}$ where $(\bar{x}, \bar{y}, \bar{z})$ are dimensional Cartesian coordinates. The interior region of the duct contains a compressible fluid, of density $\rho$ and sound speed $c$, whilst the region exterior to the duct is in vacuo. Each wall of the duct is flexible and thus able to move/vibrate in response to acoustic excitation. Under the assumption of harmonic time dependence, $e^{-i \omega \bar{t}}$, the fluid velocity potential is expressed in terms of the time independent potential by $\bar{\Phi}(\bar{x}, \bar{y}, \bar{z}, \bar{t})=\bar{\phi}(\bar{x}, \bar{y}, \bar{z}) e^{-i \omega \bar{t}}$. It is convenient to non-dimensionalise the boundary value problem with respect to length and time scales $k^{-1}$ and $\omega^{-1}$ respectively, where $\omega=c k$ and $k$ is the fluid wavenumber. Thus, non-dimensional co-ordinates are defined by $x=k \bar{x}$ etc. Similarly, $\bar{\phi}=\omega \phi / k^{2}$ etc.

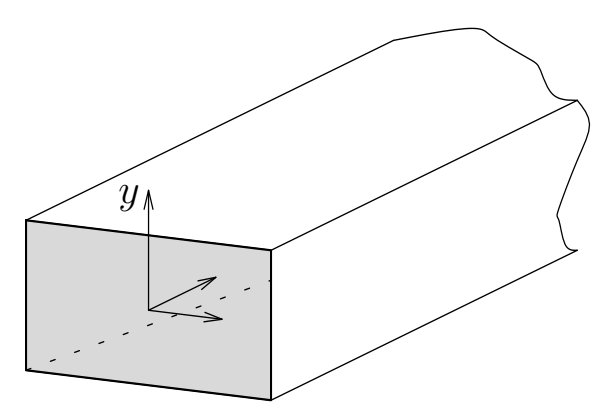

a)

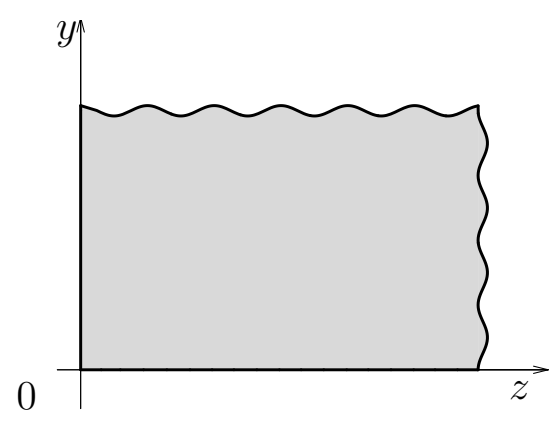

b)

Figure 1: a) the rectangular 3D duct and b) its $y z$-cross-section for $y \geq 0, z \geq 0$.

Due to coupling between the fluid and wall motions, the natural waves that travel within the duct are, in general, not separable with respect to $y$ and $z$. It can, however, be assumed that they propagate in the positive $x$ direction. The non-dimensional, timeindependent velocity potential then assumes the form

$$
\phi(x, y, z)=\sum_{n=0}^{\infty} B_{n} \Psi_{n}(y, z) e^{i s_{n} x}, \quad x>0
$$

where $B_{n}$ is the amplitude of the $\mathrm{n}^{\text {th }}$ travelling wave, $s_{n}$ is the non-dimensional axial wavenumber (assumed to be either positive real or have positive imaginary part) and the nonseparable eigenmodes $\Psi_{n}(y, z), n=0,1,2, \ldots$ are to be determined.

The modes of propagation can be symmetric with respect to both $y$ and $z$, antisymmetric with respect to both $y$ and $z$ or a combination of symmetric in $y$ (or $z$ ) and anti-symmetric in the other co-ordinate. For the sake of brevity, only fully symmetric modes will be discussed and the reader is referred to [19] where anti-symmetric modes for a duct of this class are discussed. 
For modes that are symmetric with respect to both $y$ and $z$, the eigensystem can be simplified. In this case, it is appropriate to consider wave propagation in a duct comprising two rigid walls, lying along $y=0,0 \leq z \leq b$, and $z=0,0 \leq y \leq a$ and two thin elastic plates lying along $y=a, 0 \leq z \leq b$, and $z=b, 0 \leq y \leq a$ (see figure $1 \mathrm{~b}$ ). It is convenient to treat the wavenumber as a continuous variable $s$ rather than a discrete set of values, $s_{n}$. Thus, $\Psi_{n}(y, z)=\Psi\left(s_{n}, y, z\right)$ and the potential $\Psi(s, y, z)$ satisfies reduced wave equation:

$$
\left\{\frac{\partial^{2}}{\partial y^{2}}+\frac{\partial^{2}}{\partial z^{2}}+1-s^{2}\right\} \Psi(s, y, z)=0
$$

where $0 \leq y<a, 0 \leq z \leq b$. The normal component of fluid velocity vanishes at the rigid walls which implies:

$$
\begin{aligned}
& \frac{\partial \Psi}{\partial y}=0, \quad y=0, \quad 0 \leq z \leq b \\
& \frac{\partial \Psi}{\partial z}=0, \quad z=0, \quad 0 \leq y \leq a .
\end{aligned}
$$

The plate motion is modelled using Kirchhoff theory which is valid provided $k \bar{h}<<1$ where $\bar{h}$ is the dimensional plate thickness. (For thicker plates an appropriate theory is given in [22].) Thus, the boundary condition that describes the deflections of the thin elastic plate bounding the top of the duct is

$$
\left\{\left(\frac{\partial^{2}}{\partial z^{2}}-s^{2}\right)^{2}-\mu^{4}\right\} \Psi_{y}-\alpha \Psi=0, y=a, 0 \leq z \leq b,
$$

whereas for the side wall

$$
\left\{\left(\frac{\partial^{2}}{\partial y^{2}}-s^{2}\right)^{2}-\mu^{4}\right\} \Psi_{z}-\alpha \Psi=\frac{2}{\alpha} \delta(y-a), z=b, 0 \leq y \leq a
$$

where $\delta(y-a)$ is the Dirac delta function (the presence of which is explained in section 2.2 , just below equation (18)), $\mu$ is the in vacuo plate wavenumber and $\alpha$ a fluid-loading parameter. These quantities are defined by

$$
\mu^{4}=\frac{12\left(1-\nu^{2}\right) c^{2} \rho_{p}}{k^{2} \bar{h}^{2} E} ; \quad \alpha=\frac{12\left(1-\nu^{2}\right) c^{2} \rho}{k^{3} \bar{h}^{3} E}
$$

in which $E$ is Young's modulus, $\rho$ is the density of the plate and $\nu$ is Poisson's ratio. Although attention is restricted to the situation in which the two plates are of identical material and thickness, the analysis presented herein is easily extended to the case in which the two plates have different properties.

The two elastic plates meet along the edge $y=a, z=b,-\infty<x<\infty$. Thus, in addition, to the governing equation and boundary conditions outlined above, it is necessary to apply "corner conditions" to describe how the plates are connected. It is 
assumed that the two plates are clamped to a rigid sub-frame along the edge. Thus, the appropriate corner conditions are

$$
\frac{\partial \Psi}{\partial y}=\frac{\partial \Psi}{\partial z}=\frac{\partial^{2} \Psi}{\partial y \partial z}=0, \quad y=a, \quad z=b .
$$

Following [18] and [19], an appropriate ansatz for $\Psi(s, y, z)$, can be formulated using the eigenfunctions $Y_{m}(y), m=0,1,2, \ldots$ appropriate for wave propagation in the $2 \mathrm{D}$ duct corresponding to the $x y$-cross-section of the $3 \mathrm{D}$ duct. Thus,

$$
\Psi(s, y, z)=\sum_{m=0}^{\infty} E_{m}(s) Y_{m}(y) \cosh \left[\tau_{m}(s) z\right]
$$

where the as yet unspecified quantities $E_{m}(s)$ and $\tau_{m}(s), m=0,1,2, \ldots$ depend on the parameter $s$.

\subsection{The eigensystem corresponding to the $x y$-cross-section}

Since (9) comprises an infinite sum over the functions $Y_{m}(y), m=0,1,2, \ldots$, it is expedient to define these functions and summarise their properties. These eigenfunctions characterise $2 \mathrm{D}$ acoustic propagation in a waveguide in which the upper boundary $(y=a$, $-\infty<x<\infty)$ comprises a thin elastic plate whilst the base, lying along $y=0$, $-\infty<x<\infty$, is rigid (see figure 2). Thus, the velocity potential satisfies the 2D Helmholtz's equation, with unit non-dimensional wavenumber, and the upper boundary is described by the $2 \mathrm{D}$ plate equation (that is (5) but with $\frac{\partial}{\partial z}=0$ ).

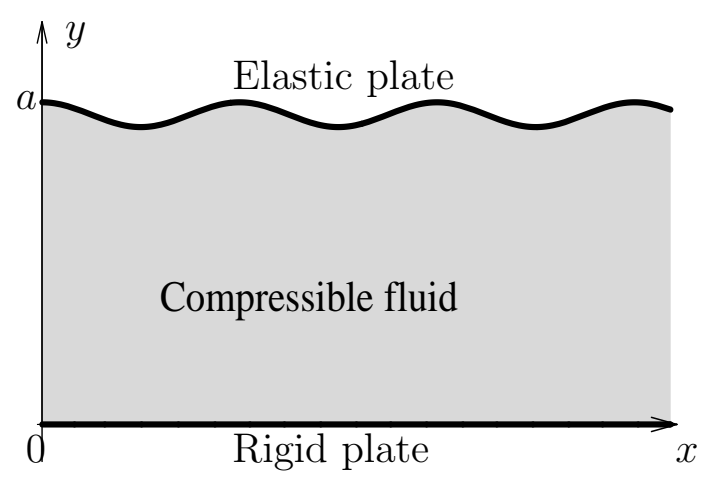

Figure 2: The $x y$-cross-section of the 3D duct.

Disturbances comprising fluid-structural waves propagating in the positive $x$-direction may be expressed in the form:

$$
\phi(x, y)=\sum_{n=0}^{\infty} A_{n} Y_{n}(y) e^{i \zeta_{n} x}, \quad x>0
$$


where $Y_{n}(y)=\cosh \left(\gamma_{n} y\right), A_{n}$ is the modal amplitude, $\zeta_{n}=\sqrt{\gamma_{n}^{2}+1}$ and is defined to be positive real or have positive imaginary part. On substituting $Y(y)=\cosh (\gamma y)$ where $\gamma=\left(\zeta^{2}-1\right)^{1 / 2}, \gamma(0)=-i$, into the plate equation, it is found that the eigenvalues $\gamma_{n}$, $n=0,1,2, \ldots$ are the roots of $K(\gamma, a)=0$ where

$$
K(\gamma, a)=\left\{\left(\gamma^{2}+1\right)^{2}-\mu^{4}\right\} \gamma \sinh (\gamma a)-\alpha \cosh (\gamma a)
$$

Here $\mu$ and $\alpha$ are the in vacuo plate wavenumber and fluid-loading parameter defined in equation (7) above. The roots of $K(\gamma, a)=0$ have the following properties: i) they occur in pairs, $\pm \gamma_{n}$; ii) there is a finite number of real roots; iii) there are infinitely many imaginary roots; iv) complex roots, $\pm \gamma_{c}$ and $\pm \gamma_{c}^{*}$ occur for some frequency ranges. The convention adopted here is that the $+\gamma_{n}$ roots are either positive real or have positive imaginary part. They are ordered sequentially, real roots first and then by increasing imaginary part. Thus, $\gamma_{0}$ is always the largest real root. It is assumed that no root is repeated. (This system of ordering is also used for the wavenumbers $s_{n}, n=0,1,2, \ldots$ in later sections.)

The eigenfunctions $Y_{m}(y), m=0,1,2, \ldots$ belong to a well studied class of function [23]. They satisfy the generalised orthogonality relation (OR):

$$
\alpha \int_{0}^{a} Y_{m}(y) Y_{j}(y) d y=C_{j} \delta_{j m}-\left(\gamma_{m}^{2}+\gamma_{j}^{2}+2\right) Y_{j}^{\prime}(a) Y_{m}^{\prime}(a)
$$

where $\delta_{j m}$ is the Kronecker delta and $C_{m}$ is given by

$$
C_{m}=\left.\frac{Y_{m}^{\prime}(a)}{2 \gamma_{m}} \frac{d}{d \gamma} K(\gamma, a)\right|_{\gamma=\gamma_{m}}
$$

in which the prime indicates differentiation with respect to $y$. They also satisfy the identities:

$$
\sum_{n=0}^{\infty} \frac{\left[Y_{n}^{\prime}(a)\right]^{2}}{C_{n}}=0, \quad \sum_{n=0}^{\infty} \frac{\gamma_{n}^{2}\left[Y_{n}^{\prime}(a)\right]^{2}}{C_{n}}=1, \quad \sum_{n=0}^{\infty} \frac{\gamma_{n}^{4}\left[Y_{n}^{\prime}(a)\right]^{2}}{C_{n}}=-2
$$

and are linearly dependent for $0 \leq y \leq a$ :

$$
\sum_{n=0}^{\infty} \frac{Y_{n}^{\prime}(a) Y_{n}(y)}{C_{n}}=\sum_{n=0}^{\infty} \frac{\gamma_{n}^{2} Y_{n}^{\prime}(a) Y_{n}(y)}{C_{n}}=0 .
$$

In addition, a Green's function can be constructed:

$$
\alpha \sum_{n=0}^{\infty} \frac{Y_{n}(v) Y_{n}(y)}{C_{n}}=\delta(y-v)+\delta(y+v)+\delta(y+v-2 a), \quad 0 \leq v, y \leq a
$$

where $\delta(y)$ is the Dirac delta function. Properties (15) and (16) prove to be very important in terms of constructing the quantity $E_{m}(s)$ of $(9)$, as is explained in the next section. 


\subsection{The $3 \mathrm{D}$ eigenfunctions}

It is clear that ansatz (9) satisfies the two rigid wall conditions and (5). On substituting (9) into the homogeneous form of (6) and bearing in mind that the $2 \mathrm{D}$ eigenfunctions are linearly dependent (15), it is seen that two eigensolutions may be constructed:

$$
\psi_{1}(s, y, z)=\sum_{m=0}^{\infty} \frac{Y_{m}^{\prime}(a) Y_{m}(y) \cosh \left[\tau_{m}(s) z\right]}{C_{m} K\left[\tau_{m}(s), b\right]}
$$

and

$$
\psi_{2}(s, y, z)=\sum_{m=0}^{\infty} \frac{\gamma_{m}^{2} Y_{m}^{\prime}(a) Y_{m}(y) \cosh \left[\tau_{m}(s) z\right]}{C_{m} K\left[\tau_{m}(s), b\right]}
$$

where $\gamma_{m}^{2}+\tau_{m}^{2}(s)-s^{2}+1=0$ and $K[\tau(s), b]$ is obtained from (11) on replacing $\gamma$ with $\tau_{m}(s)$ and $a$ with $b$. On recollecting that three corner conditions must be applied (8), it is clear that one more such solution is necessary. This is constructed by introducing a point force, in the form of a delta function, to one of the plate equations. In principle, this could be added to the right hand side of either plate equation, however, since (9) is expressed in terms of $Y_{m}(y), m=0,1,2, \ldots$, it is expedient, in view of (16), to add it to (6). Thus, on using (16) with $v=a$, the third eigensolution is found to be

$$
\chi(s, y, z)=\sum_{m=0}^{\infty} \frac{Y_{m}(a) Y_{m}(y) \cosh \left[\tau_{m}(s) z\right]}{C_{m} K\left[\tau_{m}(s), b\right]} .
$$

Thus, a solution to the eigensystem (2)-(6) is

$$
\Psi(s, y, z)=\psi_{1}(s, y, z)+P(s) \psi_{2}(s, y, z)+Q(s) \chi(s, y, z)
$$

where $P(s)$ and $Q(s)$ are arbitrary. It remains to determine $P(s), Q(s)$ and the (nondimensional) admissible wavenumbers $s_{n}, n=0,1,2, \ldots$ On applying the corner conditions (8), it is found that admissible wavenumbers are given by

$$
|\mathcal{M}(s)|=\left|\begin{array}{lll}
\psi_{1 y}(s, a, b) & \psi_{2 y}(s, a, b) & \chi_{y}(s, a, b) \\
\psi_{1 z}(s, a, b) & \psi_{2 z}(s, a, b) & \chi_{z}(s, a, b) \\
\psi_{1 y z}(s, a, b) & \psi_{2 y z}(s, a, b) & \chi_{y z}(s, a, b)
\end{array}\right|=0
$$

in which the subscripts $y$ and/or $z$ indicate differentiation with respect to these variables.

Figure 3 shows the phase speeds of the unattenuated symmetric modes as defined by (21) for the frequency range 0-1200 Hz. In order that a comparison can be made with the phase speeds of the propagating modes in a 3D rectangular duct with one flexible wall, the same parameters are used as in $[13,19]$. Thus, the duct has dimensions $\bar{a}=0.09 \mathrm{~m}$ and $\bar{b}=0.053 \mathrm{~m}$, and the plates are chosen to be aluminium, of thickness $\bar{h}=0.0006 \mathrm{~m}$ and of density $\rho_{=} 2700 \mathrm{~kg} \mathrm{~m}^{-3}$. In addition, Young's modulus and Poisson's ratio are given by 


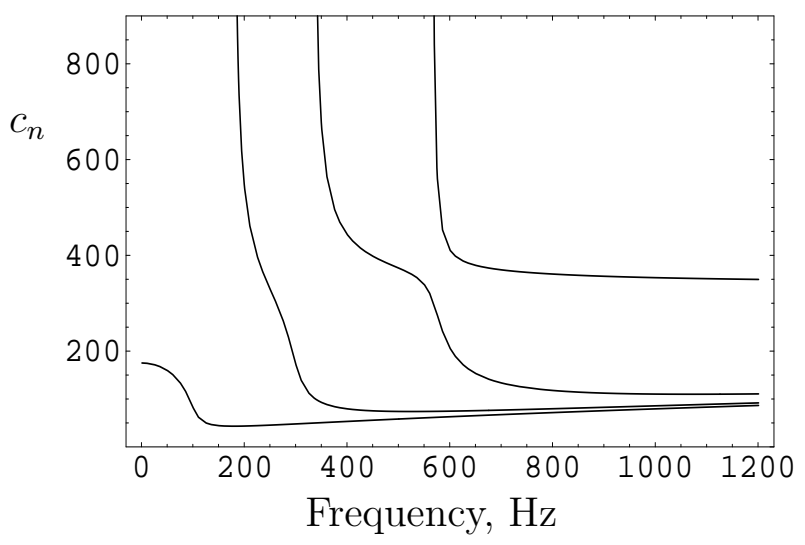

Figure 3: The phase speeds $c_{n}=c / s_{n}$ of the unattenuated symmetric waves.

$E=7.2 \times 10^{10} \mathrm{Nm}^{-2}$ and $\nu=0.34$; whilst $c$ and $\rho$ are taken to be $344 \mathrm{~ms}^{-1}$ and $1.2 \mathrm{~kg} \mathrm{~m}^{-3}$ respectively. It is clear that, for the duct considered here, there are more modes cut-on in the frequency range $0-1200 \mathrm{~Hz}$ (four as opposed to two) and that the phase speed of fundamental mode is significantly lower than for the duct comprising three rigid walls and one flexible surface $[13,19]$.

A partial orthogonality relation, similar to that presented in [18], can be constructed for the eigenmodes described above. In order to do so, however, it is necessary to specify the dispersion function in terms of the corner conditions. Here and henceforth, it is assumed that the following two corner conditions

$$
\frac{\partial \Psi}{\partial z}=\frac{\partial^{2} \Psi}{\partial y \partial z}=0, \quad y=a, \quad z=b
$$

are enforced first, thereby permitting explicit forms for $P(s)$ and $Q(s)$ to be obtained. A natural consequence is that

$$
\frac{\partial}{\partial s}\left(\frac{\partial \Psi}{\partial z}\right)=\frac{\partial}{\partial s}\left(\frac{\partial^{2} \Psi}{\partial y \partial z}\right)=0, \quad y=a, \quad z=b
$$

this result holding for all $s$. It then follows that admissible wavenumbers are the solutions to function, $L(s)=0$ where, on applying the third corner condition:

$$
L(s)=\Psi_{y}(s, a, b)
$$

The reader is reminded that, once the admissible wavenumbers are determined, the eigenmodes are given by $\Psi_{n}(y, z)=\Psi\left(s_{n}, y, z\right)$. The partial OR is derived in Appendix A and 
is stated here as:

$$
\begin{aligned}
\alpha \int_{0}^{b} \int_{0}^{a} \Psi_{n}(y, z) \Psi_{\ell}(y, z) d y d z & =D_{\ell} \delta_{\ell n} \\
- & \int_{0}^{b}\left\{\Psi_{n y y y}(a, z) \Psi_{\ell y}(a, z)\right. \\
\quad-\int_{0}^{a}\left\{\Psi_{n z z z}(y, b) \Psi_{\ell z}(y, b)\right. & \left.+\Psi_{n y}(a, z) \Psi_{\ell y y y}(y, b) \Psi_{\ell z z z}(y, b)+2 \Psi_{n z}(y, b) \Psi_{\ell z}(y, b)\right\} d y
\end{aligned}
$$

where $D_{n}=\Psi_{n y z z z}(a, b) L^{\prime}\left(s_{n}\right) /\left(2 s_{n}\right)$ with the prime indicating differentiation with respect to the argument.

It is worthwhile commenting that the eigenmodes defined by (20) are valid only for the case in which the adjacent flexible duct walls are clamped to a rigid sub-frame. In this configuration only three corner conditions are necessary. Were the pin-jointed (simply supported) case to be considered it would be necessary to add a further eigenfunction to (20). In principle this could be constructed, in a similar manner to (19), by adding a second delta function to the right hand side of (6). Bearing in mind that the eigenfunction must be symmetric with respect to $y, \delta^{\prime}(y-a)$ is inappropriate. On the other hand, if $\delta^{\prime \prime}(y-a)$ is used then $\Psi_{y z z}(y, b) \sim \delta(y-a), y \rightarrow a$ which is incompatible with the pinjointed corner conditions. It is thus not clear how to extend the general approach to case of pin-jointed corners. As will be demonstrated in section 3 , for the case of symmetry about $y=z$ only two corner conditions are needed. Hence, for a triangular duct, eigenfunctions can be constructed for clamped, pin-jointed or indeed other corner constraints.

\section{Propagation in a triangular duct}

As discussed in [19], two equivalent expressions for the symmetric eigenfunction, $\Psi(s, y, z)$, can be formulated. The first (presented above) is dependent on the eigenfunctions appropriate for wave propagation in the $2 \mathrm{D}$ duct corresponding to the $x y$-cross-section of the $3 \mathrm{D}$ duct. The alternative is similarly dependent on the eigenvalues/eigenfunctions for wave propagation in the $2 \mathrm{D}$ duct corresponding to the $x z$-cross-section. A small modification to the boundary value problem is necessary. On replacing (5) with

$$
\left\{\left(\frac{\partial^{2}}{\partial z^{2}}-s^{2}\right)^{2}-\mu^{4}\right\} \Psi_{y}-\alpha \Psi=\frac{2}{\alpha} \delta(z-b), y=a, 0 \leq z \leq b,
$$

and (6) with its homogeneous form, the solution equivalent to (20) is

$$
\Psi(s, y, z)=\tilde{\psi}_{1}(s, y, z)+\tilde{P}(s) \tilde{\psi}_{2}(s, y, z)+\tilde{Q}(s) \tilde{\chi}(s, y, z)
$$

where

$$
\tilde{\psi}_{1}(s, y, z)=\sum_{m=0}^{\infty} \frac{Z_{m}^{\prime}(b) Z_{m}(z) \cosh \left[\nu_{m}(s) y\right]}{D_{m} K\left[\nu_{m}(s), a\right]}
$$




$$
\begin{gathered}
\tilde{\psi}_{2}(s, y, z)=\sum_{m=0}^{\infty} \frac{\lambda_{m}^{2} Z_{m}^{\prime}(b) Z_{m}(z) \cosh \left[\nu_{m}(s) y\right]}{D_{m} K\left[\nu_{m}(s), a\right]}, \\
\tilde{\chi}(s, y, z)=\sum_{m=0}^{\infty} \frac{Z_{m}(b) Z_{m}(z) \cosh \left[\nu_{m}(s) y\right]}{D_{m} K\left[\nu_{m}(s), a\right]}
\end{gathered}
$$

with $\lambda_{m}^{2}+\nu_{m}^{2}(s)-s^{2}+1=0$. Here $Z_{m}(z)=\cosh \left(\lambda_{m} z\right)$ with $\lambda_{m}, m=0,1,2, \ldots$ defined by $K(\lambda, b)=0$ where $K(\lambda, b)$ is obtained from (11) on replacing $\gamma$ with $\lambda$ and $a$ with $b ; K\left[\nu_{m}(s), a\right]$ is similarly defined. This set of functions have properties analogous to (12)-(16). For example,

$$
\sum_{n=0}^{\infty} \frac{Z_{n}^{\prime}(b) Z_{n}(z)}{D_{n}}=\sum_{n=0}^{\infty} \frac{\lambda_{n}^{2} Z_{n}^{\prime}(b) Z_{n}(z)}{D_{n}}=0
$$

where

$$
D_{m}=\left.\frac{Z_{m}^{\prime}(b)}{2 \lambda_{m}} \frac{d}{d \lambda} K(\lambda, b)\right|_{\lambda=\lambda_{m}} .
$$

The two solutions (20) and (27) are entirely equivalent. In fact, it can be shown (see Appendix B) that the functions $\tilde{\psi}_{1}(s, y, z), \tilde{\psi}_{2}(s, y, z)$ and $\tilde{\chi}(s, y, z)$ can each be expressed as a linear combination of the functions $\psi_{1}(s, y, z), \psi_{2}(s, y, z)$ and $\chi(s, y, z)$. That is:

$$
\begin{aligned}
\tilde{\psi}_{1}(s, y, z) & =\psi_{1}(s, y, z) \\
\tilde{\psi}_{2}(s, y, z) & =\left(s^{2}-1\right) \psi_{1}(s, y, z)-\psi_{2}(s, y, z) \\
\tilde{\chi}(s, y, z) & =\chi(s, y, z)+\frac{\left(s^{4}-1\right)}{\alpha} \psi_{1}(s, y, z)-\frac{2\left(s^{2}+1\right)}{\alpha} \psi_{2}(s, y, z) .
\end{aligned}
$$

For the case $a=b$, a solution which is symmetric about $y=z$ can be constructed using (33)-(35). This enables the determination of admissible wavenumbers for acoustic propagation in an infinite duct of triangular cross-section and having one flexible wall (see figure 4). Symmetry about $z=0$ is assumed; thus the modes presented below also correspond to propagation in the triangular duct lying in $z \geq 0$ formed by placing a rigid wall at $z=0,0 \leq y \leq a$.

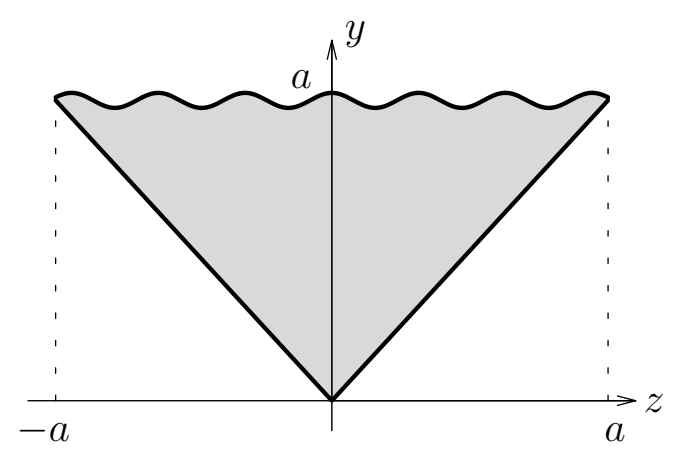

Figure 4: The $y z$-cross-section of the triangular 3-D duct. 
First it is noted that for $a=b$ the eigenvalues $\gamma_{m}$ and $\lambda_{m}, m=0,1,2, \ldots$ are equal so that $\tau_{m}(s)=\nu_{m}(s)$ and $C_{m}=D_{m}, m=0,1,2, \ldots$ Then, using (33),

$$
\psi_{1}(s, y, z)=\frac{1}{2}\left(\psi_{1}(s, y, z)+\tilde{\psi}_{1}(s, y, z)\right)=\frac{1}{2} \sum_{m=0}^{\infty} \frac{Y_{m}^{\prime}(a) J_{m}(s, y, z)}{C_{m} K\left[\tau_{m}(s), a\right]}
$$

where

$$
J_{m}(s, y, z)=\cosh \left(\gamma_{m} y\right) \cosh \left[\tau_{m}(s) z\right]+\cosh \left(\gamma_{m} z\right) \cosh \left[\tau_{m}(s) y\right]
$$

It is immediately apparent that $\nabla \psi_{1}(s, y, z) \cdot \mathbf{n}=0$ on $y=z$ where $\nabla$ denotes the gradient operator and $\mathbf{n}$ is the unit normal to the surface $y=z,-\infty<x<\infty$. Likewise,

$$
\chi(s, y, z)+\tilde{\chi}(s, y, z)=\sum_{m=0}^{\infty} \frac{Y_{m}(a) J_{m}(s, y, z)}{C_{m} K\left[\tau_{m}(s), a\right]}
$$

from which, using (35), it is apparent that $\nabla \sigma(s, y, z) \cdot \mathbf{n}=0$ on $y=z$ where

$$
\sigma(s, y, z)=\chi(s, y, z)-\frac{\left(s^{2}+1\right)}{\alpha} \psi_{2}(s, y, z) .
$$

Thus, the general solution for symmetric acoustic propagation in a right angled, isosceles triangle in which the hypotenuse comprises a thin elastic plate and the other two walls are rigid is given by

$$
\Psi(s, y, z)=\psi_{1}(s, y, z)+T(s) \sigma(s, y, z) .
$$

The coefficient $T(s)$ and the admissible wavenumbers $s_{n}, n=0,1,2, \ldots$ are determined by applying two corner conditions: in this case either clamped or pin-jointed conditions are appropriate. It transpires that for clamped corners the admissible wavenumbers are defined by $L^{c}(s)=0$ where

$$
L^{c}(s)=\psi_{1 y}(s, a, a)-\frac{\psi_{1 y z}(s, a, a)}{\sigma_{y z}(s, a, a)} \sigma_{y}(s, a, a)
$$

and for pin-jointed corners they are given by $L^{p}(s)=0$ where

$$
L^{p}(s)=\psi_{1 y}(s, a, a)-\frac{\psi_{1 y z z}(s, a, a)}{\sigma_{y z z}(s, a, a)} \sigma_{y}(s, a, a) .
$$

In (41) and (42) above the subscripts $y$ and $z$ indicate differentiation with respect to those variables. Bearing in mind both the symmetry about $y=z$ and that $\Psi_{n}(a, z)=\Psi_{n}(z, a)$, the partial OR (25) reduces to

$$
\begin{aligned}
& D_{\ell} \delta_{\ell n}=\alpha \int_{0}^{a} \int_{z}^{a} \Psi_{n}(y, z) \Psi_{\ell}(y, z) d y d z+ \\
& \quad \int_{0}^{a}\left\{\Psi_{n y y y}(a, z) \Psi_{\ell y}(a, z)+\Psi_{n y}(a, z) \Psi_{\ell y y y}(a, z)+2 \Psi_{n y}(a, z) \Psi_{\ell y}(a, z)\right\} d z
\end{aligned}
$$


where $D_{n}=\Psi_{n y z z z}(a, a) L^{c^{\prime}}\left(s_{n}\right) /\left(2 s_{n}\right)$ for clamped corners and $D_{n}=\left[\Psi_{n y z z z}(a, a)-\right.$ $\left.s_{n}^{2} \Psi_{n y z}(a, a)\right] L^{p^{\prime}}\left(s_{n}\right) /\left(2 s_{n}\right)$ for the pin-jointed case. In addition to satisfying the above relation, the eigenfunctions are linearly independent:

$$
\sum_{n=0}^{\infty} \frac{\Psi_{n y}(a, w) \Psi_{n}(y, z)}{D_{n}}=\sum_{n=0}^{\infty} \frac{\Psi_{n y y y}(a, w) \Psi_{n}(y, z)}{D_{n}}=0, \quad 0 \leq y, z \leq a
$$

and a Green's function exists:

$$
\begin{aligned}
\alpha \sum_{n=0}^{\infty} \frac{\Psi_{n}(y, z) \Psi_{n}(v, w)}{D_{n}}= & \{\delta(v-y)+\delta(v+y)+\delta(v+y-2 a)\} \\
\times & \{\delta(w+z-2 a)+\delta(z+w)+\delta(w-z)\} \\
& 0 \leq v, y, w, z \leq a .
\end{aligned}
$$

Equation (43) is a "partial" orthogonality relation in the sense that its application will not fully isolate the coefficients of an eigenfunction expansion. An integral over $z$ will remain and each term of the integrand corresponds to a viable eigensolution. The analytic properties of $\Psi_{n y}(a, z)$ and $\Psi_{n y y y}(a, z)$ ensure that a unique solution is obtained via application of suitable edge conditions. The appropriate properties are

$$
\sum_{n=0}^{\infty} \frac{\Psi_{n y}(a, w) \Psi_{n y}(a, z)}{D_{n}}=0, \quad 0 \leq w, z \leq a,
$$

and

$$
\sum_{n=0}^{\infty} \frac{\Psi_{n y y y}(a, w) \Psi_{n y}(a, z)}{D_{n}}=\delta(w+z-2 a)+\delta(-z-w)+\delta(w-z) .
$$

Properties (44)-(47) have been rigorously proved for the eigenfunctions corresponding to acoustic propagation in a $3 \mathrm{D}$ duct with three rigid walls and one flexible boundary [18]; it is anticipated that the proofs for the triangular modes considered herein would closely follow those presented in that article. For the sake of brevity the proofs are not presented here, instead the reader is offered a numerical verification of (47). Since (47) is a divergent series representing a generalised function, this cannot be performed directly. However, on multiplying both sides of $(47)$ by $f(z)$ and integrating with respect to $\mathrm{z}$, the following identity is obtained:

$$
f(w)=\int_{0}^{a}\left\{f(z) \sum_{n=0}^{\infty} \frac{\Psi_{n y y y}(a, w) \Psi_{n y}(a, z)}{D_{n}}\right\} d z .
$$

As discussed in [18], the function $f(z)$ must be even, differentiable and satisfy $f(a)=$ $f^{\prime}(a)=0$. The eigenfunctions corresponding to clamped corners are used, thus $\Psi_{n}(y, z)$ is given by (40) with $T(s)=\psi_{1 y z}(s, a, a) / \sigma_{y z}(s, a, a)$ and the admissible wavenumbers are defined by $L^{c}(s)=0$ where this quantity is given in (41). In order to verify (47) the 
left hand side and the real part of the right hand sides of (48) are plotted in figure 5 for a) $f_{1}(z)=\left(z^{2} / a^{2}-1\right)^{2} \cos (\pi z / a)$ and b) $f_{2}(z)=z \sin (\pi z / a)$. (The imaginary part of the right hand side is very small, $o\left(10^{-7}\right)$.) It is clear that the two sides of the identity are in significantly better agreement in a) than b). This is because the function plotted in b) does not satisfy the correct conditions at $z=a$. Forty terms have been used in
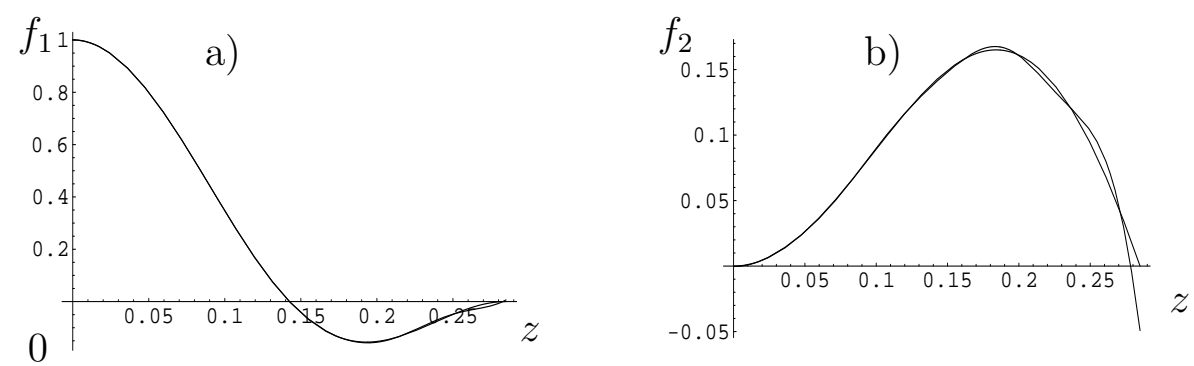

Figure 5: Numerical verification of equation (48).

the summation; increasing the number of terms will improve the accuracy of both plots, however, the discrepancy between the two curves in the vicinity of $z=a$ shown in plot $\mathrm{b}$ ) will persist. The frequency used for figure 5 is $130 \mathrm{~Hz}$ and the plate/duct parameters are stated in the next section where the use of the partial OR is demonstrated for a typical scattering problem.

\section{Scattering at the junction of two duct sections}

Two identical semi-infinite ducts of triangular cross-section lie in the regions $x<0$ and $x>0$ respectively. It is assumed that the elastic plates comprising the upper surfaces are clamped to the two rigid side walls of the triangular ducts along the corners $y=a$, $z= \pm b,-\infty<x \leq 0$ and $0 \leq x<\infty$. Hence, $\Psi_{n}(y, z)$ is given by (40) with $T(s)=$ $\psi_{1 y z}(s, a, a) / \sigma_{y z}(s, a, a)$. The fundamental structural mode with unit amplitude is incident through the left-hand duct $(x<0)$ towards $x=0$ and is scattered at the junction between the two elastic plates. There are three steps to obtaining a unique solution: first the fluid velocity potentials, $\phi_{1}(x, y, z)$ for $x<0$ and $\phi_{2}(x, y, z)$ for $x>0$, must be expressed as eigenfunction expansions in which the modal amplitudes are unknown; then the conditions of continuity of pressure and normal velocity must be imposed at the matching interface; finally suitable edge conditions must be applied at the plate junction, i.e. along the line segment $x=0, y=a, 0 \leq z \leq a$. The latter two steps are achieved by using the OR (43). Detailed summaries of the sets of edge conditions that can appropriately be applied at such a junction can be found in $[24,25,26,27]$. Two sets are considered herein: the first 
set models the situation in which the plates are pivoted at $x=0$; the second corresponds to the situation in which the plates are clamped.

It is important to note that, due to the form of the partial OR (43), only certain edge constraints are easy to apply. For example, it would be straight forward (if non-physical) to apply the condition $\phi_{1 y}(0, a, z)=\phi_{1 y y y}(0, a, z)=0$ together with $\phi_{2 y x}(0, a, z)=\phi_{2 x y y y}(0, a, z)=0$. This is because, on applying continuity of pressure and normal velocity, these quantities arise naturally in the right hand integral of (43) and can therefore be specified as zero. It is not so easy to apply the conditions corresponding to pivoted plates since these involve combinations of derivatives which do not necessarily correspond to those present in (43). A clamped junction also presents a challenge since the $\mathrm{OR}$ is not able to distinguish between, for example, $\phi_{1 y}(0, a, z)=\phi_{2 y}(0, a, z)$ and $\phi_{1 y}(0, a, z)=\phi_{2 y}(0, a, z)=0$. Thus, following [18, 27], spring-like conditions are used to impose an approximation to zero displacement and/or gradient as necessary.

The velocity potential for the fluid within the left hand duct is:

$$
\phi_{1}(x, y, z)=\Psi_{0}(y, z) e^{i s_{0} x}+\sum_{n=0}^{\infty} A_{n} \Psi_{n}(y, z) e^{-i s_{n} x}, \quad x<0
$$

where the coefficients $A_{n}, n=0,1,2, \ldots$, represent the amplitudes of the waves comprising the scattered field. Likewise, that for the right hand duct is

$$
\phi_{2}(x, y, z)=\sum_{n=0}^{\infty} B_{n} \Psi_{n}(y, z) e^{i s_{n} x}, \quad x>0 .
$$

The fluid pressure and normal velocity are both continuous at the fluid interface between the two duct sections. Continuity of pressure is expressed in terms of the modal expansions as

$$
\sum_{n=0}^{\infty}\left(A_{n}-B_{n}+\delta_{0 n}\right) \Psi_{n}(y, z)=0, \quad z \leq y \leq a, \quad 0 \leq z \leq a .
$$

On multiplying (51) by $\Psi_{\ell}(y, z)$ and integrating with respect to $y$ and $z$ it is found that:

$$
\sum_{n=0}^{\infty}\left(A_{n}-B_{n}+\delta_{0 n}\right) \int_{0}^{a} \int_{z}^{a} \Psi_{\ell}(y, z) \Psi_{n}(y, z) d y d z=0
$$

which, on using (43), implies that

$$
\begin{aligned}
\left(A_{\ell}-B_{\ell}+\delta_{0 \ell}\right) D_{\ell} & =\sum_{n=0}^{\infty}\left(A_{n}-B_{n}+\delta_{0 n}\right) \int_{0}^{a}\left\{\Psi_{n y y y}(a, z) \Psi_{\ell y}(a, z)\right. \\
& \left.+\Psi_{n y}(a, z) \Psi_{\ell y y y}(a, z)+2 \Psi_{n y}(a, z) \Psi_{\ell y}(a, z)\right\} d z .
\end{aligned}
$$

A similar procedure is followed for the equation expressing continuity of normal velocity. This is expressed in terms of the modal expansions as

$$
\sum_{n=0}^{\infty}\left(A_{n}+B_{n}-\delta_{0 n}\right) s_{n} \Psi_{n}(y, z)=0, \quad z \leq y \leq a, \quad 0 \leq z \leq a .
$$


Thus, on multiplying (54) by $\Psi_{\ell}(y, z)$ and integrating with respect to $y$ and $z$ it is found that:

$$
\sum_{n=0}^{\infty}\left(A_{n}+B_{n}-\delta_{0 n}\right) s_{n} \int_{0}^{a} \int_{z}^{a} \Psi_{\ell}(y, z) \Psi_{n}(y, z) d y d z=0
$$

which, on using (43) implies that

$$
\begin{aligned}
\left(A_{\ell}+B_{\ell}-\delta_{0 \ell}\right) s_{\ell} D_{\ell} & =\sum_{n=0}^{\infty}\left(A_{n}+B_{n}-\delta_{0 n}\right) s_{n} \int_{0}^{a}\left\{\Psi_{n y y y}(a, z) \Psi_{\ell y}(a, z)\right. \\
& \left.+\Psi_{n y}(a, z) \Psi_{\ell y y y}(a, z)+2 \Psi_{n y}(a, z) \Psi_{\ell y}(a, z)\right\} d z
\end{aligned}
$$

As discussed above, the edge conditions are now used to simplify equations (53) and (56).

\subsection{Pivoted junction}

In this section the first of the two sets of edge conditions mentioned above is considered. That is, the two plates are pivoted along $x=0, y=a, 0 \leq z \leq b$. The appropriate edge conditions are zero displacement for the left hand plate:

$$
\phi_{1 y}(0, a, z)=0, \quad 0 \leq z \leq b
$$

together with continuous gradient:

$$
\phi_{1 y x}(0, a, z)=\phi_{2 y x}(0, a, z), \quad 0 \leq z \leq b
$$

In addition, the bending moment is continuous:

$$
\phi_{1 y x x}(0, a, z)+\nu \phi_{1 y z z}(0, a, z)=\phi_{2 y x x}(0, a, z)+\nu \phi_{2 y z z}(0, a, z), \quad 0 \leq z \leq b
$$

and a vertical spring condition is applied to the right-hand plate

$$
\phi_{2 y x x x}(0, a, z)+(2-\nu) \phi_{2 x y z z}+\beta \phi_{2 y}(0, a, z)=0,0 \leq z \leq a
$$

Bearing in mind (57) and that $\phi_{j}(x, y, z), j=1,2$ and its first derivatives satisfy the the 3D Helmholtz equation with unit wavenumber, (59) may be written as:

$$
\phi_{1 y y y}(0, a, z)-\phi_{2 y y y}(0, a, z)=(1-\nu) \phi_{2 y z z}(0, a, z)+\phi_{2 y}(0, a, z), \quad 0 \leq z \leq b
$$

whilst the spring condition can be similarly rearranged as

$$
\phi_{2 y x y y}(0, a, z)-(1-\nu) \phi_{2 x y z z}+\phi_{2 y x}=\beta \phi_{2 y}(0, a, z), 0 \leq z \leq a .
$$

Note that, as $\beta \rightarrow \infty$ (62) tends to the required zero displacement condition. 
On using the condition of zero displacement for the left hand plate, the pressure condition (53) reduces to

$$
\begin{aligned}
A_{\ell} & =-\delta_{0 \ell}+B_{\ell}-\frac{1}{D_{\ell}} \sum_{n=0}^{\infty} B_{n}\left[U_{\ell n}+2 V_{n \ell}\right] \\
& +\frac{1}{D_{\ell}} \sum_{n=0}^{\infty}\left(A_{n}-B_{n}+\delta_{0 n}\right) \int_{0}^{a} \Psi_{n y y y}(a, z) \Psi_{\ell y}(a, z) d z
\end{aligned}
$$

Equation (61) can be cast in modal form as:

$$
\sum_{n=0}^{\infty}\left(A_{n}-B_{n}+\delta_{0 n}\right) \Psi_{n y y y}(a, z)=\sum_{n=0}^{\infty} B_{n}\left[(1-\nu) \Psi_{n y z z}(a, z)+\Psi_{n y}(a, z)\right], \quad 0 \leq z \leq b
$$

and thus (63) reduces to:

$$
A_{\ell}=-\delta_{0 \ell}+B_{\ell}+\frac{1}{D_{\ell}} \sum_{n=0}^{\infty} B_{n}\left[(1-\nu) W_{n \ell}-V_{n \ell}-U_{\ell n}\right]
$$

where

$$
\begin{aligned}
U_{n \ell} & =\int_{0}^{a} \Psi_{n y y y}(a, z) \Psi_{\ell y}(a, z) d z \\
V_{n \ell} & =\int_{0}^{a} \Psi_{n y}(a, z) \Psi_{\ell y}(a, z) d z, \\
W_{n \ell} & =\int_{0}^{a} \Psi_{n y z z}(a, z) \Psi_{\ell y}(a, z) d z=\left(s_{n}^{2}-1\right) V_{n \ell}-U_{n \ell} .
\end{aligned}
$$

In a similar manner, continuity of plate gradient at $x=0$ reduces (56) to

$$
B_{\ell}=\delta_{0 \ell}-A_{\ell}+\frac{1}{s_{\ell} D_{\ell}} \sum_{n=0}^{\infty}\left(A_{n}+B_{n}-\delta_{0 n}\right) s_{n} \int_{0}^{a} \Psi_{n y y y}(a, z) \Psi_{\ell y}(a, z) d z
$$

It remains to enforce the spring condition. This may be expressed as

$$
\sum_{n=0}^{\infty} B_{n} s_{n} \Psi_{n y y y}(a, z)=\sum_{n=0}^{\infty} B_{n} s_{n}\left[(1-\nu) \Psi_{n y z z}(a, z)-\Psi_{n y}(a, z)\right]-i \beta \sum_{n=0}^{\infty} B_{n} \Psi_{n y}(a, z)
$$

and, on substituting (70) into (69), it is found that

$$
\begin{aligned}
B_{\ell}=\delta_{0 \ell}-A_{\ell} & +\frac{1}{s_{\ell} D_{\ell}} \sum_{n=0}^{\infty}\left(A_{n}-\delta_{0 n}\right) s_{n} U_{n \ell} \\
& +\frac{1}{s_{\ell} D_{\ell}} \sum_{n=0}^{\infty} B_{n} s_{n}\left[(1-\nu) W_{n \ell}-V_{n \ell}\right]-\frac{i \beta}{s_{\ell} D_{\ell}} \sum_{n=0}^{\infty} B_{n} V_{n \ell}
\end{aligned}
$$

Thus, the coefficients $A_{\ell}$ and $B_{\ell}$ are determined by numerically solving (65) together with (71). 
Figure 6 shows the real and imaginary parts of the scaled dimensional plate displacement $\bar{\eta}(\bar{x}, \bar{z}) / 4$ where $\bar{\eta}(\bar{x}, \bar{z})=i k^{-1} \phi_{y}(\bar{x}, \bar{a}, \bar{z})$, at a frequency of $130 \mathrm{~Hz}$ (for which the dispersion relation has one real root) and with $\beta=30000000$. (A high value of $\beta$ has been chosen to ensure that the right hand plate $(x>0)$ displacement is effectively zero.) The dimension height of the duct at its vertex is $\bar{a}=0.12 \mathrm{~m}$ whilst the total plate width is $2 \bar{a}=0.24 \mathrm{~m}$. The plate is aluminium, of thickness $\bar{h}=0.0006 \mathrm{~m}$ and of density $\rho=2700$ $\mathrm{kg} \mathrm{m}^{-3}$. In addition, Young's modulus and Poisson's ratio are given by $E=7.2 \times 10^{10}$ $\mathrm{Nm}^{-2}$ and $\nu=0.34$; whilst $c$ and $\rho$ are taken to be $344 \mathrm{~ms}^{-1}$ and $1.2 \mathrm{~kg} \mathrm{~m}^{-3}$ respectively. The reader is reminded that the plate displacement displayed herein, although scaled by a factor of 0.25 , is grossly exaggerated - the forcing amplitude would, in reality, be very small. It is clear that the incoming wave undergoes both reflection and transmission at the plate junction $(\bar{x}=0)$. Some transmission is to be expected since, although both plate displacements are zero (or near zero), the plate gradient is non-zero and continuous. Thus, the motion on the right hand plate arises both due to fluid-structure interaction and the continuity of plate gradient. Two (unscaled) plate sections are shown for $\bar{z}=\bar{a} / 2$, $-0.1 \leq \bar{x} \leq 0.1$. These are included as a pictorial verification that the edge conditions on each plate have been satisfied.

a)

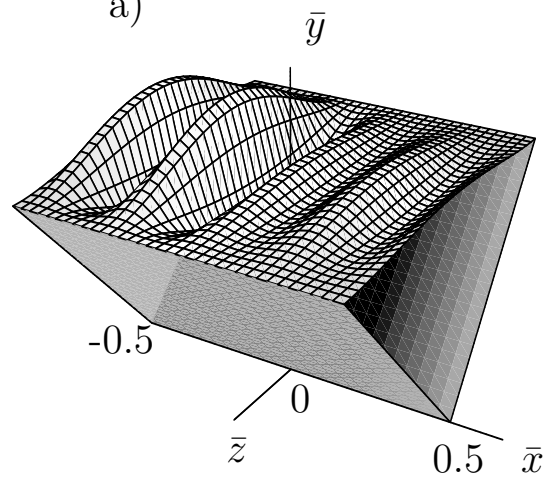

b)

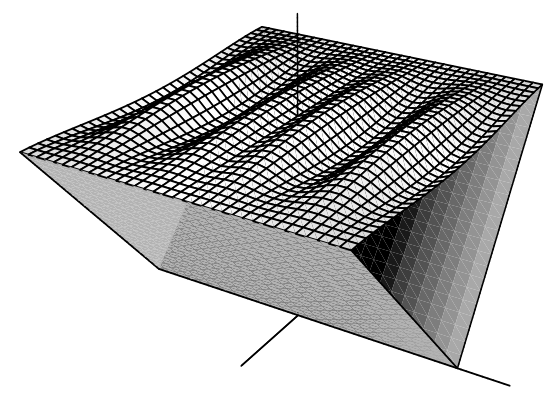

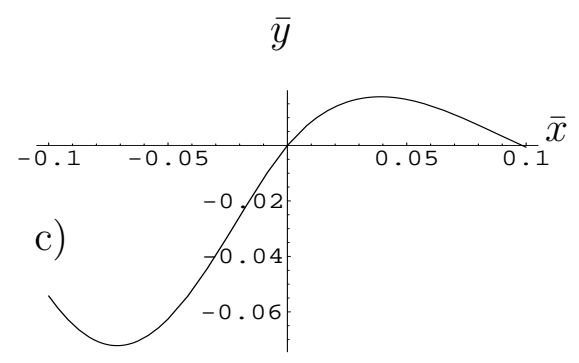

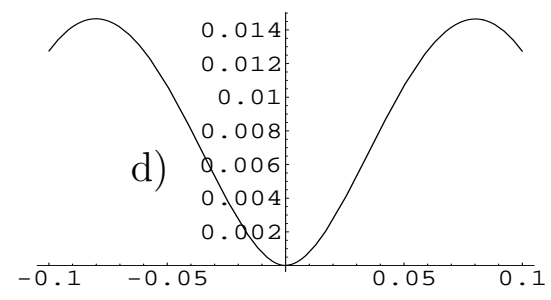

Figure 6: Pivoted junction: plate displacement at $130 \mathrm{~Hz}$; a) and b) real and imaginary parts, $-0.5 \leq \bar{x} \leq 0.5$; c) and d) cross-section of real and imaginary parts at $\bar{z}=\bar{a} / 2$, $-0.1 \leq \bar{x} \leq 0.1$. 


\subsection{Clamped junction}

As mentioned above, the OR is unable to distinguish between continuous edge conditions $\phi_{1 y}(0, a, z)=\phi_{2 y}(0, a, z)$ and zero edge conditions $\phi_{1 y}(0, a, z)=\phi_{2 y}(0, a, z)=0$. Thus, approximate conditions, which tend to the required conditions in a specified limit, are used. It follows that the edge conditions for the left hand plate are zero displacement

$$
\phi_{1 y}(0, a, z)=0, \quad 0 \leq z \leq a
$$

and a condition which closely corresponds [27] to a rotational spring

$$
\phi_{1 y x x}(0, a, z)+\nu \phi_{1 y z z}(0, a, z)+\beta_{1} \phi_{1 y x}=0, \quad 0 \leq z \leq a .
$$

On noting (72) and using Helmholtz's equation, this reduces to:

$$
\phi_{1 y y y}(0, a, z)=\beta_{1} \phi_{1 y x}, \quad 0 \leq z \leq a .
$$

For the right hand plate the appropriate conditions are zero gradient

$$
\phi_{2 y x}(0, a, z)=0, \quad 0 \leq z \leq a
$$

and the vertical spring condition which, on using (75) in (62), is

$$
\phi_{2 y x y y}(0, a, z)-(1-\nu) \phi_{2 x y z z}=\beta_{2} \phi_{2 y}(0, a, z), 0 \leq z \leq a .
$$

The rotational spring condition for the left hand plate implies

$$
\sum_{n=0}^{\infty}\left(A_{n}+\delta_{0 n}\right) \Psi_{n y y y}(a, z)=-i \beta_{1} \sum_{n=0}^{\infty}\left(A_{n}-\delta_{0 n}\right) s_{n} \Psi_{n y}(a, z) .
$$

On applying zero plate displacement (72) and (77) the right hand side of (53) simplifies and it is found that

$$
A_{\ell}=-\delta_{0 \ell}+B_{\ell}-\frac{1}{D_{\ell}} \sum_{n=0}^{\infty} B_{n} R_{n \ell}-\frac{i \beta_{1}}{D_{\ell}} \sum_{n=0}^{\infty}\left(A_{n}-\delta_{0 n}\right) s_{n} V_{n \ell}
$$

where

$$
R_{n \ell}=2 V_{n \ell}+U_{n \ell}+U_{\ell n} .
$$

On applying the zero gradient condition (75) together with the vertical spring condition (56) reduces to

$$
\begin{aligned}
B_{\ell}=\delta_{0 \ell}-A_{\ell} & +\frac{1}{s_{\ell} D_{\ell}} \sum_{n=0}^{\infty}\left(A_{n}-\delta_{0 n}\right) s_{n} R_{n \ell} \\
& +\frac{(1-\nu)}{s_{\ell} D_{\ell}} \sum_{n=0}^{\infty} B_{n} s_{n} W_{n \ell}-\frac{i \beta_{2}}{s_{\ell} D_{\ell}} \sum_{n=0}^{\infty} B_{n} V_{n \ell} .
\end{aligned}
$$


Thus, the coefficients $A_{\ell}$ and $B_{\ell}$ are determined by numerically solving (78) together with (80).

Figure 7 shows the real and imaginary parts of the scaled dimensional plate displacement $\bar{\eta}(\bar{x}, \bar{z}) / 4$ where $\bar{\eta}(\bar{x}, \bar{z})=i k^{-1} \phi_{y}(\bar{x}, \bar{a}, \bar{z})$, at a frequency of $130 \mathrm{~Hz}$ and with $\beta_{1}=\beta_{2}=30000000$. (High values have been chosen to ensure that the both plates are effectively clamped.) It is clear that the incoming wave is largely reflected at the plate junction $(\bar{x}=0)$ - arguably more so than for the pivoted junction. This is to be expected since the edge conditions dictate that both plate displacements and gradients are zero (or near zero). Thus, the motion on the right hand plate arises primarily due to fluid-structure interaction. Two (unscaled) plate sections are shown for $\bar{z}=\bar{a} / 2,-0.1 \leq \bar{x} \leq 0.1$; these verify that the edge conditions on each plate have been satisfied.

a)

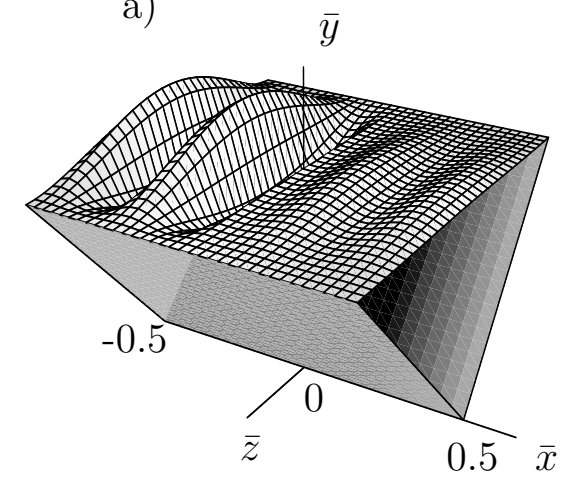

b)

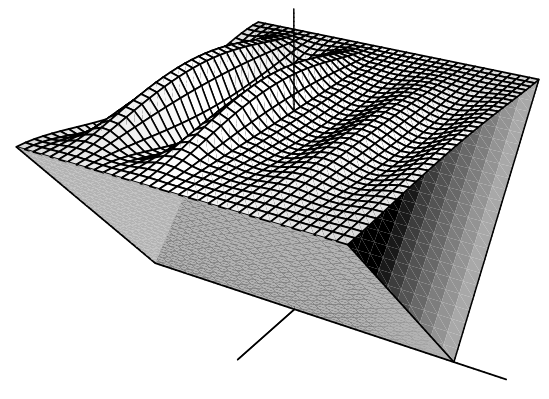

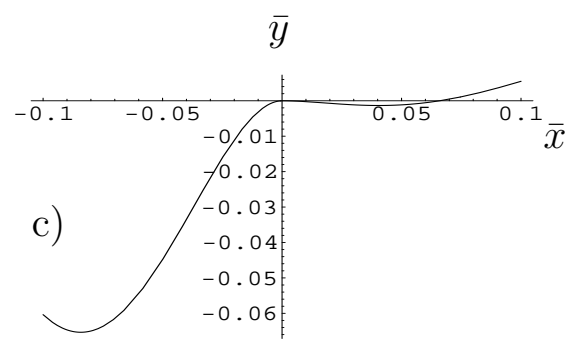

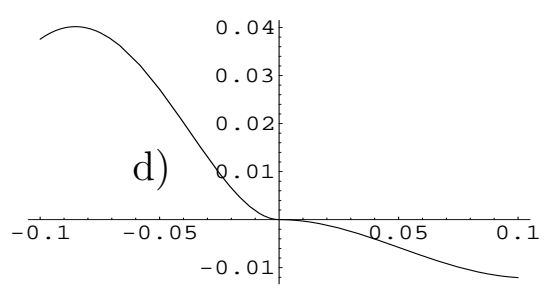

Figure 7: Clamped junction: plate displacement at 130Hz; a) and b) real and imaginary parts, $-0.5 \leq \bar{x} \leq 0.5$; c) and d) cross-section of real and imaginary parts at $\bar{z}=\bar{a} / 2$, $-0.1 \leq \bar{x} \leq 0.1$.

\section{Discussion}

The eigenfunctions for acoustic propagation in a rectangular duct with four flexible walls have been presented. Two equivalent forms for these eigenmodes were derived: the first is dependent on the eigenvalues for the underlying $2 \mathrm{D}$ problem corresponding to the 
$x y$-cross-section of the 3D duct; the second is analogously dependent on those for the $x z$-cross-section. Knowledge of these two forms enables the construction of the eigenmodes for a right-angled, isosceles triangular duct in which the hypotenuse is flexible. The properties of the triangular modes have been discussed and used to develop an analytic mode-matching method appropriate for problems involving the scattering of fluidstructural coupled waves in 3D ducts with flexible walls. Mode-matching solutions for a typical scattering problem have been presented for two distinct sets of junction conditions.

The eigenmodes, (20) and (40), considered herein comprise a number of infinite sums and, as mentioned above, each of these involves the eigenvalues corresponding to propagation in the underlying $2 \mathrm{D}$ duct which must be determined numerically. Due to the presence of the quantity $K\left[\tau_{m}(s), b\right]$ in the denominator, each sum is highly convergent and can be truncated after relatively few terms. For example, the phase speeds presented in figure 3 were computed using 35 terms in the expressions for $\psi_{1}(s, y, z), \psi_{2}(s, y, z)$, $\chi(s, y, z)$ etc. and for the numerical results of section 4 these sums were evaluated using 58 terms. However, an undesirable consequence of the presence of $K\left[\tau_{m}(s), b\right]$ in the denominator of the sums is that $\Psi_{n}(y, z) \rightarrow 0$ and $D_{n} \rightarrow 0$ as $n \rightarrow \infty$. For this reason, the triangular eigenfunctions were rescaled before numerical implementation. A factor of $s^{4}$ was removed from the term $K\left[\tau_{m}(s), a\right]$ in the denominator, after which $\Psi_{n}(y, z) / \sqrt{D_{n}}$ was used as opposed to $\Psi_{n}(y, z)$. This rescaling offers two advantages: firstly it reduces the problems associated with computing very small numbers; secondly, it provides a means of assessing the accuracy to which $\Psi_{n}(y, z) / \sqrt{D_{n}}$ is calculated. That is, since the left hand side of (43) is rescaled simply to $\delta_{n \ell}$, any significant deviation of the right hand side from one when $n=\ell$ suggests that accuracy is a problem.

Another potential source of error is, of course, the root-finding. Two sets of roots must be found: the $2 \mathrm{D}$ eigenvalues $\gamma_{n}, n=0,1,2, \ldots$ and the $3 \mathrm{D}$ wavenumbers, $s_{n}$, $n=0,1,2, \ldots$ Fortunately, the argument principle provides a relatively simple check by which to confirm that all the required the $2 \mathrm{D}$ eigenvalues have been found and this set of roots seldom presents a problem. With regard to the 3D characteristic equations, the presence of poles as well as zeros means that the argument principle is less helpful. Furthermore, the zeros often lie very close to the poles - a point which is discussed more fully in [19]. The identity (48) is, however, very sensitive to missing roots and, when used at a single point, provides a suitable check for the 3D wavenumbers. Nevertheless, should a large number of roots be required, the root-finding process can prove time consuming.

The analytic mode-matching scheme developed herein was used to derive two infinite systems of linear equations, (65) together with (71) and (78) together with (80), corresponding to the solution of a typical scattering problem for two different sets of "junction" conditions. These were truncated and solved numerically. The "acid-test" of any mode- 
matching scheme is the accuracy to which the continuity conditions are satisfied at the matching interface. In order gain an idea as to how well the left and right hand normal velocities agree these quantities (calculated using (49) and (50)) were compared along a number arbitrary horizontal lines $(y=2 a / 3, y=a / 2, y=a / 3)$ within the matching interface. For both the clamped and pivoted junctions, at $130 \mathrm{~Hz}$ and using $40 \mathrm{terms}$, the relative error was consistently less than $2 \%$ - although did this did tend to increase for values of $y$ closer to the right angled corner. It is worth noting that the edge conditions used in section 5.2 correspond to a clamped junction as $\beta_{1}, \beta_{2} \rightarrow \infty$ but in the limit $\beta_{1} \rightarrow 0, \beta_{2} \rightarrow \infty$ they correspond to a junction in which the left hand plate is pin-jointed (simply supported) and the right hand plate is clamped. It was found that, although the edge conditions were well satisfied, the normal velocities exhibited a higher relative error for the pin-jointed/clamped junction. The significant difference here is that the plate gradients are not continuous/zero. It appears that the system of equations is slower to converge in such cases. In all cases, however, the pressures and velocity fluxes are in excellent agreement.

As far as the author is aware, the analytic mode-matching scheme presented herein is the first available for ducts with flexible walls and non-separable eigenfunctions. The approach is equally appropriate for the triangular duct considered in sections 3 and 4 or for rectangular ducts with four flexible walls, although in the latter case the appropriate partial OR is (25). It is worth noting that the method also has application in the field of hydrodynamics. For example, when the effects of surface tension are included, the freesurface boundary conditions are of high order (equivalent to a membrane) and thus nonseparable waveforms for a 3D wave-maker of triangular cross-section can be formulated in an analogous manner to those presented in section 3. Another potential application is the study of wave propagation in ice-covered channels of triangular cross-section. In these contexts the fluid-wall or ice-wall contact conditions replace the corner conditions relevant to this study. Although some contact conditions lead to separable eigenfunctions $[20,21]$ others will give rise to non-separable modes. The article by Harter et al.[28] contains a good discussion of some plausible contact conditions which could, in principle, be incorporated into the theory in a similar manner to the clamped or pin-jointed corner constraints discussed herein. 


\section{A Derivation of the partial orthogonality relation}

In this appendix the partial orthogonality relation (25) is derived using equations (2)-(6). From (3) and (5) it may be seen that

$$
\left[\left\{\left(\frac{\partial^{2}}{\partial z^{2}}-s^{2}\right)^{2}-\mu^{4}\right\} \Psi_{n y} \Psi_{\ell y}-\alpha \Psi_{n} \Psi_{\ell y}\right]_{y=0}^{y=a}=0 .
$$

On interchanging the subscripts $\ell$ and $n$ and subtracting the resulting expression from (A.81), it is found that

$$
\alpha \int_{0}^{a}\left(\Psi_{n} \Psi_{\ell y y}-\Psi_{\ell} \Psi_{n y y}\right) d y-\left[G_{\ell n}(y, z)\right]_{y=0}^{y=a}=0
$$

where

$$
\begin{aligned}
G_{\ell n}(y, z) & =\Psi_{n y z z z z} \Psi_{\ell y}-\Psi_{\ell y z z z z} \Psi_{n y}-2 s_{n}^{2} \Psi_{n y z z} \Psi_{\ell y}+2 s_{\ell}^{2} \Psi_{\ell y z z} \Psi_{n y} \\
& +\left(s_{n}^{4}-s_{\ell}^{4}\right) \Psi_{n y} \Psi_{\ell y} .
\end{aligned}
$$

A similar expression can be constructed using (4) and (6), that is

$$
\alpha \int_{0}^{b}\left(\Psi_{n} \Psi_{\ell z z}-\Psi_{\ell} \Psi_{n z z}\right) d z-\left[F_{\ell n}(y, z)\right]_{z=0}^{z=b}=0
$$

where

$$
\begin{aligned}
F_{\ell n}(y, z) & =\Psi_{n z y y y y} \Psi_{\ell z}-\Psi_{\ell z y y y y} \Psi_{n z}-2 s_{n}^{2} \Psi_{n z y y} \Psi_{\ell z}+2 s_{\ell}^{2} \Psi_{\ell z y y} \Psi_{n z} \\
& +\left(s_{n}^{4}-s_{\ell}^{4}\right) \Psi_{n z} \Psi_{\ell z}-\frac{2}{\alpha} \delta(y-a)\left(\Psi_{\ell z}-\Psi_{n z}\right) .
\end{aligned}
$$

On integrating (A.82) with respect to $z, 0 \leq z \leq b$ and (A.84) with respect to $y, 0 \leq y \leq a$; adding the two results together and then using (2) to simplify the integrand, it is found that

$$
\alpha \int_{0}^{b} \int_{0}^{a} \Psi_{n}(y, z) \Psi_{\ell}(y, z) d y d z=\frac{1}{\left(s_{\ell}^{2}-s_{n}^{2}\right)}\left\{\int_{0}^{b} G_{\ell n}(a, z) d z+\int_{0}^{a} F_{\ell n}(y, b) d y\right\} .
$$

For the case $\ell \neq n$ repeated integration by parts yields

$$
\begin{aligned}
\int_{0}^{b} G_{\ell n}(a, z) d z & =-\left(s_{\ell}^{2}-s_{n}^{2}\right) \int_{0}^{b}\left[2 \Psi_{n y z}(a, z) \Psi_{\ell y z}(a, z)\right. \\
& \left.+\left(s_{n}^{2}+s_{\ell}^{2}\right) \Psi_{n y}(a, z) \Psi_{\ell y}(a, z)\right] d z
\end{aligned}
$$

A similar expression is obtained for the integral on the right hand side of (A.86) containing $F_{\ell n}(y, b)$. Hence, for $m \neq n$

$$
\begin{aligned}
\alpha & \int_{0}^{b} \int_{0}^{a} \Psi_{n}(y, z) \Psi_{\ell}(y, z) d y d z= \\
& -\int_{0}^{b}\left[2 \Psi_{n y z}(a, z) \Psi_{\ell y z}(a, z)+\left(s_{n}^{2}+s_{\ell}^{2}\right) \Psi_{n y}(a, z) \Psi_{\ell y}(a, z)\right] d z \\
& -\int_{0}^{a}\left[2 \Psi_{n y z}(y, b) \Psi_{\ell y z}(y, b)+\left(s_{n}^{2}+s_{\ell}^{2}\right) \Psi_{n z}(y, b) \Psi_{\ell z}(y, b)\right] d y
\end{aligned}
$$


For $\ell=n$ it is necessary to interpret the right hand side of (A.86) carefully. The first step to to replace $G_{\ell n}(a, z)$ and $F_{\ell n}(y, b)$ with $G_{n}(s, a, z)$ and $F_{n}(s, y, b)$ respectively, where the latter quantities are obtained on replacing every occurrence of $\Psi_{\ell}(y, z)$ (and its derivatives) with $\Psi(s, y, z)$ (and the appropriate derivatives). Note, that $\Psi_{n}(y, z)$ and its derivatives are not altered. Then,

$$
\begin{aligned}
& \alpha \int_{0}^{b} \int_{0}^{a} \Psi_{n}(y, z) \Psi_{n}(y, z) d y d z= \\
& \lim _{s \rightarrow s_{n}} \frac{1}{2 s}\left\{\int_{0}^{b} \frac{\partial}{\partial s} G_{n}(s, a, z) d z+\int_{0}^{a} \frac{\partial}{\partial s} F_{n}(s, y, b) d y\right\} .
\end{aligned}
$$

On differentiating with respect to $s$ and taking the limit $s \rightarrow s_{n}$, it is found that

$$
\begin{aligned}
\left.\frac{1}{2 s_{n}} \int_{0}^{b} \frac{\partial}{\partial s} G_{n}(s, a, z)\right|_{s=s_{n}} d z= & \\
-2 \int_{0}^{b}\left[\Psi_{n y z}(a, z) \Psi_{n y z}(a, z)\right. & \left.+s_{n}^{2} \Psi_{n y}(a, z) \Psi_{n y}(a, z)\right] d z \\
+\frac{1}{2 s_{n}}\left[\left[\Psi_{n y z z z}-2 s_{n}^{2} \Psi_{n y z}\right)\right] \frac{\partial}{\partial s} \Psi_{y}(s) & \left.-\Psi_{n y z z} \frac{\partial}{\partial s} \Psi_{y z}(s)+\Psi_{n y z} \frac{\partial}{\partial s} \Psi_{y z z}(s)\right]_{s=s_{n}}
\end{aligned}
$$

where the $s$ dependence is shown but it is understood that the functions are evaluated at $y=a, z=b$. A similar expression is obtained for the last term of (A.89) and on recalling (23), it is deduced that

$$
\begin{aligned}
& \alpha \int_{0}^{b} \int_{0}^{a} \Psi_{n}(y, z) \Psi_{\ell}(y, z) d y d z=D_{\ell} \delta_{\ell n} \\
& \quad-\int_{0}^{b}\left[2 \Psi_{n y z}(a, z) \Psi_{\ell y z}(a, z)+\left(s_{n}^{2}+s_{\ell}^{2}\right) \Psi_{n y}(a, z) \Psi_{\ell y}(a, z)\right] d z \\
& \quad-\int_{0}^{a}\left[2 \Psi_{n y z}(y, b) \Psi_{\ell y z}(y, b)+\left(s_{n}^{2}+s_{\ell}^{2}\right) \Psi_{n z}(y, b) \Psi_{\ell z}(y, b)\right] d y
\end{aligned}
$$

where for clamped corners $D_{n}=\Psi_{n y z z z}(a, b) L^{\prime}\left(s_{n}\right) /\left(2 s_{n}\right)$ with the prime indicating differentiation with respect to the argument, whilst for pin-jointed corners $D_{n}=\left[\Psi_{n y z z z}(a, b)-\right.$ $\left.2 s_{n}^{2} \Psi_{n y z}(a, b)\right] L^{\prime}\left(s_{n}\right) /\left(2 s_{n}\right)$. Finally, on integrating by parts and using (2) to rearrange the integrand, expression (25) is obtained.

\section{B Proof of expression (35)}

Result (35) is proved by examining the families of poles arising in the integrand of $I(s)=0$ where

$$
I(s, y, z)=\lim _{\mathcal{T} \rightarrow \infty} \frac{1}{2 \pi i} \int_{-\mathcal{T}}^{\mathcal{T}} \frac{\cosh (\lambda b) \cosh (\lambda z) \cosh [\nu(s) y] d \lambda}{K(\lambda, b) K[\nu(s), a] \sinh (\lambda b)} .
$$

Here $\nu(s)=\left(s^{2}-1-\lambda^{2}\right)^{1 / 2}$ and the path of integration lies along the real axis and is indented above(below) any singularities on the negative(positive) section. There is one 
pole at $\lambda=0$ and the residue contribution from this is interpreted in the spirit of a Cauchy principal value integral. That is, the average of the values that would be obtained on taking the contour to lie a) above and b) below this point is taken - this amounts to half the usual residue contribution. There are three families of poles; the residue contributions arising due to these are denoted by $R_{j}(s, y, z), j=1,2,3$. Then, on deforming the path of integration onto a semi-circular arc of radius $\mathcal{T}$ in the upper half plane and noting that there is no contribution from the arc as $\mathcal{T} \rightarrow \infty$, Cauchy's residue theorem gives

$$
R_{1}(s, y, z)+R_{2}(s, y, z)+R_{3}(s, y z)=0
$$

where the residue contributions from each infinite set of poles are discussed below.

The first set of poles is defined by $K(\lambda, b)=0$. Thus $\lambda=\lambda_{m}, m=0,1,2, \ldots$. On using (32), the residue contributions can be expressed as

$$
R_{1}(s, y, z)=\sum_{m=0}^{\infty} \frac{\cosh \left(\lambda_{m} b\right) \cosh \left(\lambda_{m} z\right) \cosh [\nu(s) y]}{\left.\frac{d}{d \lambda} K(\lambda, b)\right|_{\lambda=\lambda_{m}} K\left[\nu_{m}(s), a\right] \sinh \left(\lambda_{m} b\right)}=\frac{1}{2} \tilde{\chi}(s, y, z) .
$$

The second family of poles corresponds to $K[\nu(s), a]=0$. Thus, they occur when $\nu(s)=\gamma_{m}$ and $\lambda=\tau_{m}(s), m=0,1,2, \ldots$ and it follows that

$$
R_{2}(s, y, z)=\sum_{m=0}^{\infty} \frac{\operatorname{coth}\left[\tau_{m}(s) b\right] \cosh \left(\gamma_{m} y\right) \cosh \left[\tau_{m}(s) z\right]}{\left.K\left[\tau_{m}(s), b\right] \frac{d}{d \lambda} K\left[\nu_{m}(s), a\right]\right|_{\lambda=\tau_{m}(s)}} .
$$

It is easily confirmed that

$$
\left.\frac{d}{d \lambda} K\left[\nu_{m}(s), a\right]\right|_{\lambda=\tau_{m}(s)}=-\left.\frac{\tau_{m}(s)}{\gamma_{m}} \frac{d}{d \gamma} K(\gamma, a)\right|_{\gamma=\gamma_{m}}
$$

and so, on using (13):

$$
R_{2}(s, y, z)=-\frac{1}{2} \sum_{m=0}^{\infty} \frac{Y_{m}^{\prime}(a) Y_{m}(y) \cosh \left[\tau_{m}(s) z\right]}{\tau_{m}(s) \tanh \left[\tau_{m}(s) b\right] K\left[\tau_{m}(s), b\right] C_{m}} .
$$

This expression is then rearranged in two steps: first (11) is used to express $1 /\left(\tau_{m}(s) \tanh \left[\tau_{m}(s) b\right]\right)$ in terms of $K\left[\tau_{m}(s), b\right]$; then the quantity $\gamma_{m}^{4}-\mu^{4}$ (which arises as a result of step one) is eliminated by noting that $K\left(\gamma_{m}, a\right)=0$ (again see (11)). It thus transpires that

$$
\begin{aligned}
R_{2}(s, y, z) & =-\frac{1}{2} \chi(s, y, z)-\frac{\left(s^{4}-1\right)}{2 \alpha} \psi_{1}(s, y, z)+\frac{\left(s^{2}+1\right)}{\alpha} \psi_{2}(s, y, z) \\
& +\frac{1}{2 \alpha} \sum_{m=0}^{\infty} \frac{Y_{m}^{\prime}(a) Y_{m}(y) \cosh \left[\tau_{m}(s) z\right]}{\tau_{m}(s) \sinh \left[\tau_{m}(s) b\right] C_{m}} .
\end{aligned}
$$

The final set of poles is given by $\lambda \sinh (\lambda b)=0$ and thus occur when $\lambda=i m \pi / b$, $m=0,1,2, \ldots$. It is found that

$$
R_{3}(s, y, z)=-\frac{1}{\alpha b} \sum_{m=0}^{\infty} \frac{(-1)^{m} \cosh \left[\nu_{m}(s) y\right] \cos (m \pi z / b)}{\varepsilon_{n} K\left[\nu_{m}(s), a\right]}
$$


where $\varepsilon_{n}=2$ if $n=0$ and 1 otherwise. The presence of this quantity in the denominator of (B.99) arises, as discussed above, due to the fact that the first residue in this family is located at $\lambda=0$.

On substituting (B.94), (B.98) and (B.99) into (B.93), it is found

$$
\begin{aligned}
0 & =\frac{1}{2} \tilde{\chi}(s, y, z)-\frac{1}{2} \chi(s, y, z)-\frac{\left(s^{4}-1\right)}{2 \alpha} \psi_{1}(s, y, z)+\frac{\left(s^{2}+1\right)}{2 \alpha} \psi_{2}(s, y, z) \\
& +\frac{1}{2 \alpha} \sum_{m=0}^{\infty} \frac{\left.Y_{m}^{\prime}(a) Y_{m}(y) \cosh \left[\tau_{m}(s) z\right)\right]}{\tau_{m}(s) \sinh \left[\tau_{m}(s) b\right] C_{m}} \\
& -\frac{1}{\alpha b} \sum_{m=0}^{\infty} \frac{(-1)^{m} \cosh \left[\nu_{m}(s) y\right] \cos (m \pi z / b)}{\varepsilon_{n} K\left[\nu_{m}(s), a\right]}
\end{aligned}
$$

The last two terms comprise two different representations of the same eigenfunction (that for acoustic propagation in a 3D rectangular duct with one flexible wall) and thus cancel. This point is discussed in [19] and may also be proved using a similar analysis to that above. Hence, (B.100) reduces to (35). Results (33) and (34) can be proved using the same approach.

\section{References}

[1] I.D. Abrahams, Scattering of sound by finite elastic surfaces bounding ducts or cavities near resonance, Q J Mech Appl Math 35(1) (1982) 91-101.

[2] A. Cabelli, The propagation of sound in a square duct with a non-rigid wall, J. Sound Vib. 103 (1985) 379-394.

[3] L. Huang, Modal analysis of a drumlike silencer, J. Acoust. Soc. Am. 112 (2002) 2014-2025.

[4] R. Kirby, Modeling sound propagation in acoustic waveguides using a hybrid numerical method, J. Acoust. Soc. Am.124(4) (2008) 1930-1940.

[5] R. Kirby, A comparison between analytic and numerical methods for modelling automotive dissipative silencers with mean flow, J. Sound Vib. 325 (2009) 565-582.

[6] J.B. Lawrie, R. Kirby "Mode-matching without root-finding: application to a dissipative silencer", J. Acoust. Soc. Am. 119, 2050-2061 (2006).

[7] B. Nennig, E. Perrey-Debain, M. Ben Tahar, A mode matching method for modeling dissipative silencers lined with poroelastic materials and containing mean flow, J. Acoust. Soc. Am. 128 (2010) 3308-3320 . 
[8] W. Bi, V. Pagneux, D. Lafarge, Y. Aurégan, An improved multimodal method for sound propagation in nonuniform lined ducts, J. Acoust. Soc. Am. 122 (2007) 280-290.

[9] P.A. Martin, The horn-feed problem: sound waves in a tube joined to a cone, and related problems, J. Eng. Math 71 (2011) 291-304.

[10] J.B. Lawrie, I.D. Abrahams, An orthogonality condition for a class of problems with high order boundary conditions; applications in sound/structure interaction, Q. Jl Mech appl. Math. 52 (1999) 161-181.

[11] J.B. Lawrie, Comments on a class of orthogonality relations relevant to fluid-structure interaction, Meccanica 47(3) (2012) 783-788.

[12] L. Huang, Y.S. Choy, Vibroacoustics of three-dimensional drum silencer, J. Acoust. Soc. Am. 118 (2005) 2313-2320.

[13] V. Martin, A. Cummings, C. Gronier, Discrimination of coupled structural/acoustical duct modes by active control: principles and experimental results, J. Sound Vib. 274 (2004) 583-603.

[14] R.J. Astley, A. Cummings, N. Sormaz, A finite element scheme for acoustic propagation in flexible walled ducts with bulk-reacting liners, and comparison with experiment, J. Sound Vib. 150 (1991) 119-138.

[15] G. Gabard, R.J. Astley, A computational mode-matching approach for sound propagation in three-dimensional ducts with flow, J. Sound Vib. 315 (2008) 1103-1124.

[16] B. Venkatesham, M. Tiwari, M.L. Munjal, Analytical prediction of the breakout noise from a rectangular cavity with one compliant wall, J. Acoust. Soc. Am. 124 (2008) 29522962.

[17] B. Venkatesham, M. Tiwari, M.L. Munjal, Analytical prediction of the break-out noise from a reactive rectangular plenum with four flexible walls, J. Acoust. Soc. Am. 128 (2010) 1789-1799.

[18] J.B. Lawrie, Orthogonality relations for fluid-structural waves in a 3-D rectangular duct with flexible walls, Proc. R. Soc. A., 465 (2009) 2347-2367.

[19] J.B. Lawrie, On acoustic propagation in three-dimensional rectangular ducts with flexible walls and porous linings, J. Acoust. Soc. Am. 131(3) (2012) 1890-1901. 
[20] R. Mondal, S.K. Mohanty, T. Sahoo, Expansion formulae for wave structure interaction problems in three dimensions, IMA J Appl Math (2012) doi:10.1093/imamat/hxr044.

[21] R. Mondal, T. Sahoo, Wave structure interaction problems for two-layer fluids in three dimensions, Wave Motion 49 (2012) 501-524.

[22] J.D. Kaplunov, E.V. Nolde, B.F. Shorr, A perturbation approach for evaluating natural frequencies of moderately thick elliptic plates, J. Sound Vib. 281 (2005) 905-919.

[23] J.B. Lawrie, On eigenfunction expansions associated with wave propagation along ducts with wave-bearing boundaries, IMA J. Appl. Math. 72 (2007) 376-394.

[24] P.R. Brazier-Smith, The acoustic properties of two co-planar half-plane plates, Proc. R. Soc. A. 409 (1987) 115-139.

[25] A.N. Norris, G.R. Wickham, Acoustic diffraction from the junction of two flat plates, Proc. R. Soc. A. 451 (1995) 631-655.

[26] J.B. Lawrie, I.D. Abrahams, Scattering of fluid loaded elastic plate waves at the vertex of a wedge of arbitrary angle, I: analytic solution, IMA J Appl Math 59 (1997) $1-23$.

[27] H. Chung, C. Fox, A direct relationship between bending waves and transition conditions of floating plates, Wave Motion 46 (2009) 468-479.

[28] R. Harter, M.J. Simon, I.D. Abrahams, The effect of surface tension on localized freesurface oscillations about surface-piercing bodies, Proc. R. Soc. A, 464 (2008) 3039-3054. 